\title{
The impact of SUZAKU in Frontier Astrophysics
}

\author{
Hideyuki Mori ${ }^{* a b}$, and the Suzaku team \\ a CRESST and X-ray Astrophysics Laboratory, NASA Goddard Space Flight Center, Greenbelt, \\ MD 20771, USA \\ ${ }^{b}$ Department of Physics, University of Maryland, Baltimore County, 1000 Hilltop Circle, \\ Baltimore, MD 21250, USA \\ E-mail: hideyuki.morienasa.gov
}

We present here outstanding observational results obtained with Suzaku, the Japanese 5th X-ray satellite. Since new findings explored by Suzaku cover a wide category of the X-ray astronomy, we pick up three topics in the present paper in my personal point of view. The topics are summarized as follows: (1) Clear detection of emission line of the C VI Ly $\gamma$ due to the interaction between the solar-wind ions and $\mathrm{H}$ I atom in the geocorona, (2) Discovery of the radiative recombination continua in the X-ray spectrum of the supernova remnant, IC 443, indicating that the presence of overionized plasma, and (3) Exploration of the physical properties of hot intracluster medium beyond the virial radius of a cluster of galaxies, Perseus cluster. Such spectroscopic studies will make great progress by an X-ray micro-calorimeter with energy resolution of $6 \mathrm{eV}$ at $6 \mathrm{keV}$ onboard the next Japanese X-ray mission, ASTRO-H.

Frontier Research in Astrophysics,

26-31 May 2014

Mondello (Palermo), Italy

\footnotetext{
* Speaker.
} 


\section{Introduction - the Japanese X-ray satellite Suzaku}

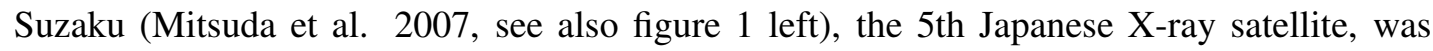
launched in July, 2005. For imaging spectroscopy to cover the $0.2-12 \mathrm{keV}$ range, this satellite equips an X-ray micro-calorimeter (XRS; Kelley et al. 2007) and four X-ray CCD cameras in combination with X-ray Telescopes (XRT; Serlemitsos et al. 2007). The XRS is a non-dispersive detector to achieve high energy resolution of $\sim 6 \mathrm{eV}$ at $6 \mathrm{keV}$ by measuring a temperature change of the X-ray absorber. Hence, it was expected to exploit plasma diagnostics for diffuse X-ray emission, such as clusters of galaxies and supernova remnants, using the resonance, intercombination, and forbidden lines from highly ionized (He-like) ions. However, due to the leakage of liquid He to cool the absorber down to $60 \mathrm{mK}$, the XRS had been unusable unfortunately soon after the launch.

Meanwhile, the X-ray CCD cameras (XIS; Koyama et al. 2007) have characteristics of moderately good energy resolution $(\sim 130 \mathrm{eV}$ at $6 \mathrm{keV})$ and low Non X-ray Background (NXB). Since Suzaku was placed in a near-circular orbit with altitude of $\sim 550 \mathrm{~km}$ and inclination angle of $31 \mathrm{de}-$ gree, the particle background below $10 \mathrm{keV}$ is sufficiently low. Figure $\square$ right shows the comparison of the background levels between some X-ray missions. This low and stable background allows us to detect diffuse X-ray emission with low surface brightness efficiently. The XIS also has capability of spaced-row charge injection (SCI); periodic artificial charges input to the imaging area mitigate the charge transfer inefficiency due to the radiation damage. Thanks to the SCI technique, the degradation of the energy gain and resolution has been somewhat suppressed.

Suzaku also possesses the non-imaging Hard X-ray Detector (HXD; Takahashi et al. 2007) consisting of Si-PIN diodes and GSO scintillators to cover the 10-600 keV band. The HXD enables us to obtain wide-band spectra for studies of non-thermal phenomena in the universe.
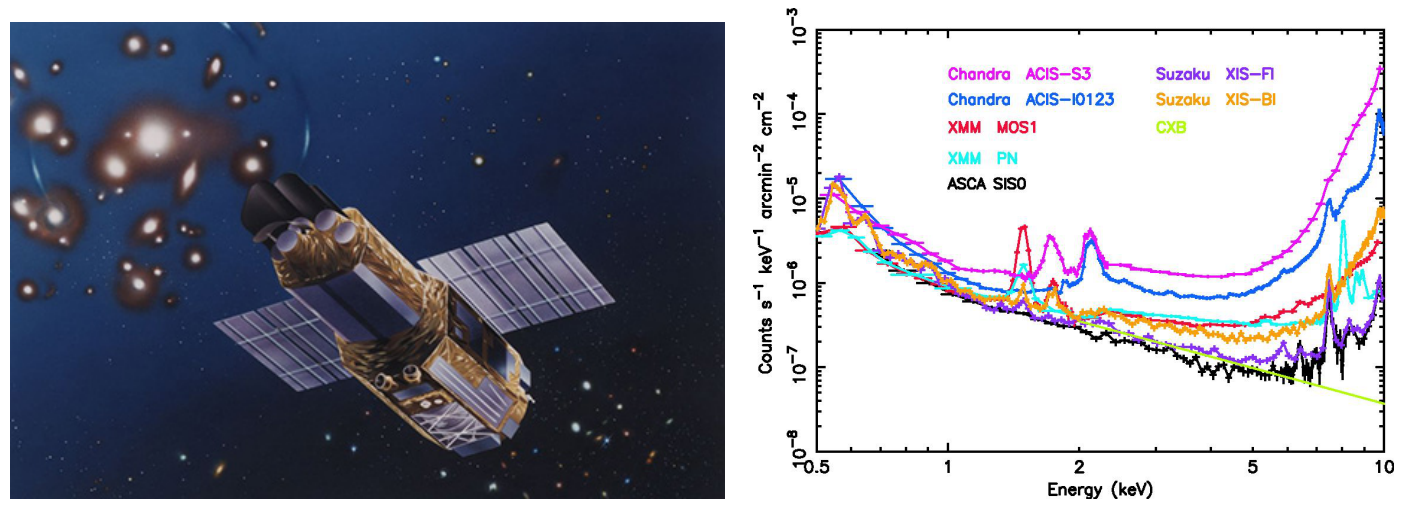

Figure 1: (Left) Imaginary picture of Suzaku in orbit (CISAS/JAXA). (Right) Detector backgrounds of some X-ray missions. This figure is adapted from Mitsuda et al. (2007).

\section{Results}

Many new findings have been acquired through the Suzaku observations so far, and then have given impacts on the X-ray astronomy in this 9 years. Since the results obtained with Suzaku cover a wide range of celestial targets, we cannot provide a comprehensive review in this paper. Thus, we would like to focus on the outstanding topics, picked up from my personal point of view.

\subsection{Solar Wind Charge Exchange}

Using the X-ray optics, ROSAT All-Sky Survey (RASS, Truemper 1982) had revealed the entire sky in soft X-rays (Snowden et al. 1995). While diffuse X-ray emission along the Galactic 
plane at $1 / 4 \mathrm{keV}$ was hampered by the heavy absorption of the interstellar medium (ISM), the Xray maps at $3 / 4 \mathrm{keV}$ and $1.5 \mathrm{keV}$ showed local enhancements that reflect the distribution of the hot gas in the Galaxy, e.g., Local Hot Bubble or Loop I. In the RASS, Snowden et al. (1994) reported a mysterious $\mathrm{X}$-ray variability in the R1/R2 bands with a timescale of 1-2 days. A key to understand the origin of this long-term variability was the discovery of the X-ray emission from comets (e.g., Dennerl et al. 1997); the emission mechanism was found to be charge exchange between solarwind ions and neutral atoms (Cravens 1997). The solar-wind charge exchange with $\mathrm{H}$ atoms in the Earth's geocorona and in the heliosphere was then suggested to explain the soft X-ray background below $1 \mathrm{keV}$ (e.g., Cravens 2000). Robertson et al. (2001) indicated a correlation between the time variability in the RASS data and the solar-wind proton flux, suggesting the existence of the charge exchange in the geocorona. With the advent of Chandra and XMM-Newton, the spectral information on the solar-wind charge exchange became available as line emissions.

To investigate the cosmic soft X-ray background, the foreground components consisting of diffuse emission originated from the hot gas in the Galactic plane and halo should be comprehensively understood. The sporadic contamination due to the solar-wind charge exchange in the geocorona has hindered this attempt; the quantitative determination of its contribution to the soft X-ray emission is essential to elucidate the physical condition of the Galactic hot ISM. Suzaku allows us to perform the detailed spectroscopy for the solar-wind charge exchange.

Fujimoto et al. (2007) reported the Suzaku observation of a blank sky towards the North Ecliptic Pole (NEP). The authors found that the X-ray count rate in the $0.3-2 \mathrm{keV}$ band increased during $\sim 4 \times 10^{4}$ s from the observation start. This X-ray enhancement cannot be explained with the variation of the XIS NXB. The X-ray image and spectrum in the $0.2-2 \mathrm{keV}$ range during the enhancement are shown in figure $\square$. The authors compared the spectrum with that obtained in the time interval with a stable X-ray count rate, and then found that the enhancement was represented with an increase in the emission lines, probably originated from ionized $\mathrm{C}, \mathrm{O}, \mathrm{Ne}, \mathrm{Mg}$, and $\mathrm{Fe}$ ions, and the power-law tail. Among the identification of the lines, the $n=4$ to 1 transition of $\mathrm{C}$ VI $(459 \mathrm{eV})$ was needed for the detected line at $455 \mathrm{eV}$. Although the $\mathrm{C}$ VI Ly $\gamma$ emission due to the charge exchange was already detected from the Mars observation with XMM-Newton (Dennerl et al. 2006), the NEP observation firstly revealed that such an interaction should be occurred as well in the Earth's magnetosheath at 2-8 Earth radii.
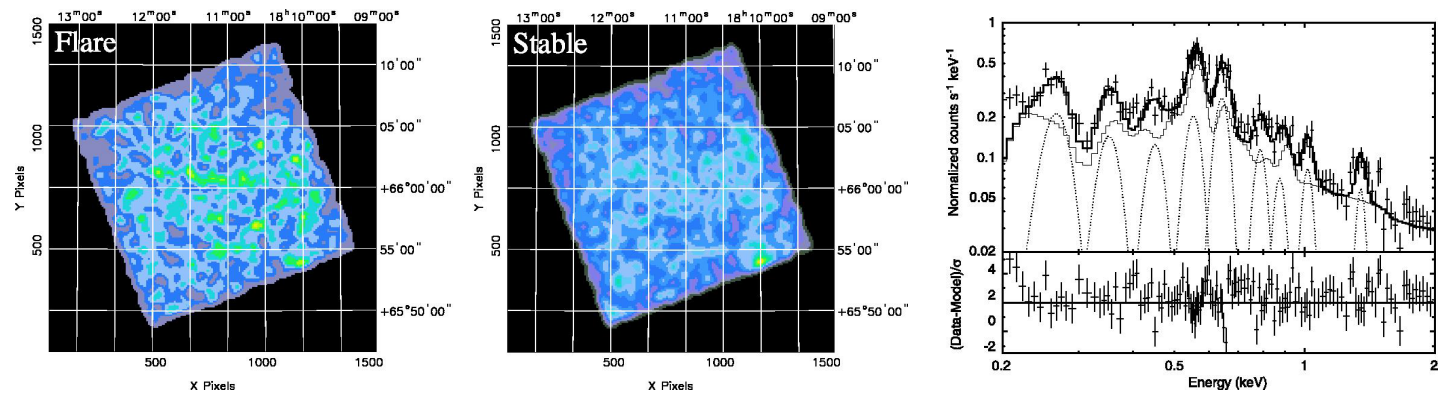

Figure 2: (Left) Suzaku XIS images of the NEP region. (Right) XIS spectrum in the soft X-ray enhancement. These figure are adapted from Fujimoto et al. (2007).

\subsection{Overionized plasma in supernova remnants (SNRs)}

Supernovae are one of the most energetic phenomena in the universe. Ejecta from progenitor 
stars expands with a velocity of $\sim 10^{4} \mathrm{~km} \mathrm{~s}^{-1}$. Thus, a blast wave is formed. The blast wave heats the ejecta and its surrounding ISM up to $\sim 10^{7} \mathrm{~K}$, and then these materials are ionized to be hot plasma, which emits X-rays with bremsstrahlung. The electron temperature $\left(T_{\mathrm{e}}\right)$ can be derived from optically-thin thermal emission in X-ray spectra. The collisional interaction between the nuclei and electrons makes He-like or $\mathrm{H}$-like ions that have a large population in the plasma at $\sim 10^{7} \mathrm{~K}=1 \mathrm{keV}$. These highly-ionized ions show many characteristic emission lines.

The emission lines in X-ray spectra provide a powerful tool to examine the physical condition of this thermal plasma. The intensity ratio of the $\mathrm{K} \alpha$ and $\mathrm{K} \beta$ emission lines originated from the same ionized species depends on the electron temperature. Meanwhile, we can estimate the ionization temperature $\left(T_{\mathrm{i}}\right)$ that represents the ion population using the intensity ratio of the Helike and $\mathrm{H}$-like emission lines. According to a theoretical model to the time evolution of the SNR plasma, soon after the shock heating, the plasma is not yet in thermal and ionization equilibrium; the ion temperature $\left(T_{\text {ion }}\right)$ is significantly larger than $T_{\mathrm{e}}$. Energy transfers via Coulomb collision increase the electron temperature, and then hot electrons gradually proceed the ionization. Hence, $T_{\text {ion }} \gg T_{\mathrm{e}}>T_{\mathrm{i}}$ holds in the non-equilibrium ionization (NEI) plasma.

Contrary to this normal evolution for young SNRs, Kawasaki et al. (2002) found that the Xray emitting plasma of IC 443 (Jellyfish nebula) was likely to be overionized $\left(T_{\mathrm{i}}>T_{\mathrm{e}}\right)$, utilizing the $\mathrm{K} \alpha$ emission lines of He-like and H-like S in the ASCA SIS spectrum. While IC 443 shows the shell-like emission at radio/infrared/optical wavelengths, indicating the SNR interaction with the dense molecular cloud, the X-ray emission is center-filled in the shell. Troja et al. (2006) claimed that their spectral result obtained with XMM-Newton suggested the X-ray emitting plasma in near equilibrium, different from the result of Kawasaki et al. (2002).

Yamaguchi et al. (2009) solved this problem. The authors reported that the Suzaku spectra of IC 443 cannot be fully reproduced with the bremsstrahlung continuum and the emission lines. They found that the spectra required some additional continua representing the radiative recombination process for S, Si, Mg (Radiative Recombination Continuum; RRC, see figure (B)); due to the freebound transition of electrons, triangle-shaped continuum proportional to $\exp \left(-\frac{E-I}{k T_{\mathrm{e}}}\right)$ is emerged $(E$ and $I$ represent the electron and bound-state energies, respectively). This spectroscopic finding of RRC strongly supports that this radiative-recombination dominated plasma is indeed overionized.
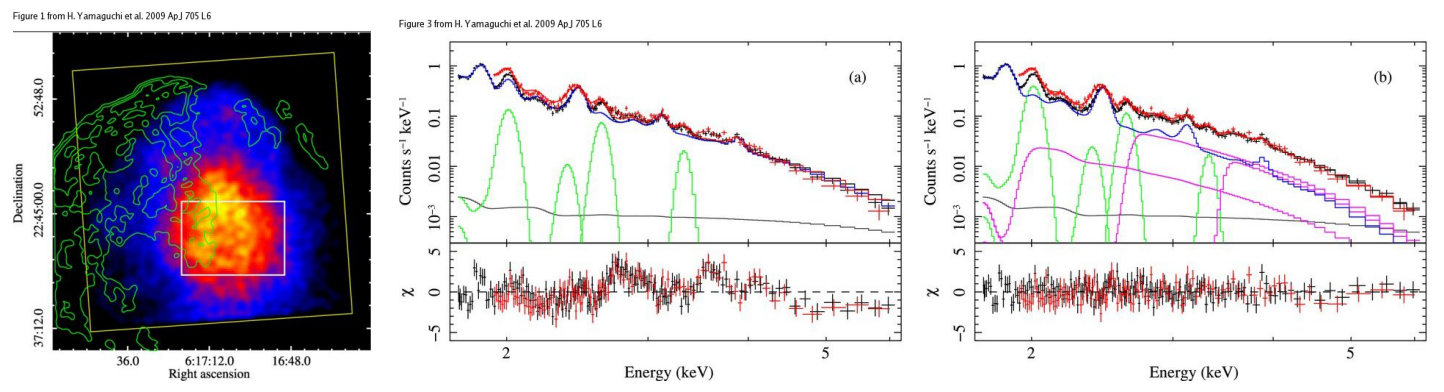

Figure 3: (Left) Suzaku XIS image of IC443. (Right) XIS spectra of IC 443 without and with some radiative recombination continua. These figures are adapted from Yamaguchi et al. (2009)

\subsection{Outskirts of cluster of galaxies}

A cluster of galaxies is one of the largest structure in the universe that is gravitationallybounded system with a typical mass of $\sim 10^{14} M_{\odot}$. X-ray observations of clusters of galaxies 
had unveiled that these systems are ubiquitously filled with optically-thin thermal plasma with temperature of $10^{7}-10^{8} \mathrm{~K}$ (e.g. Sarazin 1988), and that member galaxies observed at optical and infrared wavelengths have only a small fraction of the baryon mass in the system; the kinematics of the member galaxies indicates the presence of a deep gravitational well, $\gtrsim 100$ times larger than that formed with these galaxies. Assuming the isothermality and hydrostatic equilibrium of the hot intracluster medium (ICM), which can be observed only with X-rays, the total mass of the cluster is estimated. Clusters of galaxies thus prove the existence of dark matter in the universe. Furthermore, a large fraction of baryons has not yet been observed directly (dark baryon). We note that the search of the dark baryon is planned extensively; one of the attempts is the Japanese DIOS mission (Ohashi et al. 2014) to detect by X-ray micro-calorimeter redshifted O VII and O VIII emission lines from "Warm-Hot Intergalactic Medium (WHIM)" with $T \sim 10^{5}-10^{6} \mathrm{~K}$.

The physical properties of the ICM are key probes to elucidate the history of the dynamical formation of the large-scale structure. However, the detailed spectroscopic studies of the ICM has been so far limited to the bright inner part of the cluster; the outskirts of the cluster are too faint to be examined. Thanks to the large collecting area of the XRTs and the low/stable background level of the XIS, the Suzaku observation, for the first time, has approached the cluster outskirts beyond to the virial radius. Simionescu et al. (2011) carried out the mapping observation of the Perseus cluster, as one of the Suzaku Key Projects. Figure $\mathbb{G}$ shows the X-ray mosaic image of the Perseus cluster. Their finding is that the outskirts of the cluster of galaxies is not static but dynamic. The right panel of figure $⿴$ 9 shows the radial profiles of electron density, entropy and pressure. The deviations from the expected profiles are found at $r>2 / 3 r_{\mathrm{vir}}\left(r_{\mathrm{vir}}\right.$ : virial radius). The simple assumption of the gas in hydrostatic equilibrium is no longer correct to this extended regions. Hence, this result indicates that the mass estimation should be changed from that was used to be. The deviation from the hydrostatic equilibrium is theoretically expected to be caused by some mechanisms: gas bulk motion, turbulent, magnetic fields, cosmic-rays, and so on. To make clear the gas dynamics, the next Japanese X-ray mission, ASTRO-H, is expected.
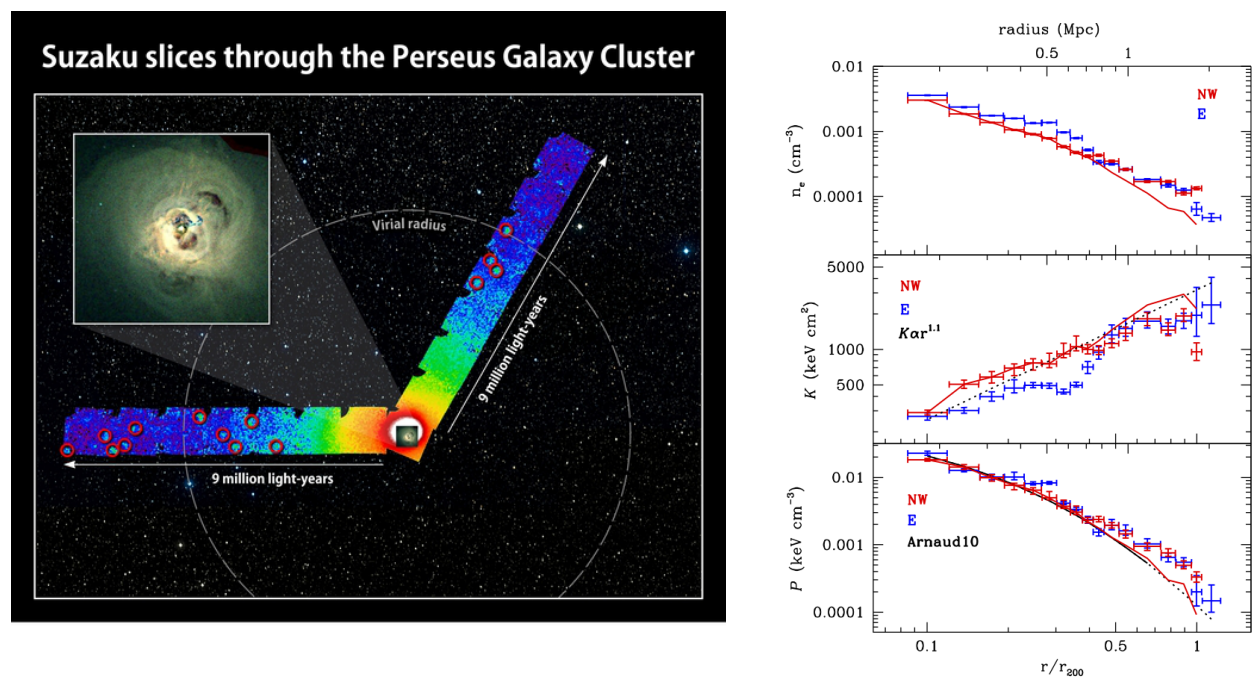

Figure 4: (Left) Suzaku mosaic image of Perseus cluster. (Right) Radial profiles of the electron density, entropy, and pressure. These figures are adapted from http://www.astro.isas.ac.jp/suzaku/flash/2011/0325/ (C)ISAS/JAXA) and Simionescu et al. (2011), respectively 


\section{Conclusion}

Spectroscopic studies are the basic style of the Japanese X-ray group; the imaging spectroscopy above $2 \mathrm{keV}$ had been achieved by the X-ray CCD cameras and nested thin-foil mirror onboard ASCA (Tanaka et al. 1994), and then was inherited by Suzaku. In spite of the XRS malfunction, all of the topics presented here was achieved based on plasma diagnostics utilizing the XRT+XIS spectroscopy.

Now, we are developing the next X-ray satellite, ASTRO-H (Takahashi et al. 2012). ASTROH equips an X-ray micro-calorimeter (SXS) for the re-challenge of the Suzaku XRS, which will be operated with the He tank and Joule-Thomson cooler during $>3$ years. ASTRO-H also has a widefield X-ray CCD camera $\left(38^{\prime} \times 38^{\prime}\right)$. Moreover, the Hard X-ray Telescopes and Hard X-ray Imager are employed to perform imaging spectroscopy in the $5-80 \mathrm{keV}$ band. In combination of these instruments, high-resolution and wide-band spectra are expected to evolve the plasma diagnostics for clusters of galaxies and SNRs as well as X-ray point sources such as active stars, magnetic white dwarf, neutron-star or black-hole binaries. ASTRO-H will be launched in 2016.

Acknowledgments I would like to thank all the Suzaku and ASTRO-H team members for their dedication to X-ray astronomy.

\section{References}

[1] Cravens, T. E. 1997, Geophysical Research Letters, 24, 105

[2] Cravens, T. E. 2000, ApJL, 532, L153

[3] Cravens, T. E. 2002, Science, 296, 1042

[4] Dennerl, K., Englhauser, J., \& Trümper, J. 1997, Science, 277, 1625

[5] Dennerl, K., Lisse, C. M., Bhardwaj, A., et al. 2006, A\&A, 451, 709

[6] Kawasaki, M. T., Ozaki, M., Nagase, F., et al. 2002, ApJ, 572, 897

[7] Kelley, R. L., Mitsuda, K., Allen, C. A., et al. 2007, PASJ, 59, 77

[8] Koyama, K., et al. 2007, PASJ, 59, 23

[9] Mitsuda, K., Bautz, M., Inoue, H., et al. 2007, PASJ, 59, 1

[10] Ohashi, T., Ishisaki, Y., Ezoe, Y., et al. 2014, Proc. SPIE, 9144, 91442Q

[11] Robertson, I. P., Cravens, T. E., Snowden, S., \& Linde, T. 2001, Space Sci. Rev., 97, 401

[12] Sarazin, C. L. 1988, Cambridge Astrophysics Series, Cambridge: Cambridge University Press, 1988,

[13] Serlemitsos, P. J., et al. 2007, PASJ, 59, 9

[14] Simionescu, A., Allen, S. W., Mantz, A., et al. 2011, Science, 331, 1576

[15] Snowden, S. L., Freyberg, M. J., Plucinsky, P. P., et al. 1995, ApJ, 454, 643

[16] Takahashi, T., Mitsuda, K., Kelley, R., et al. 2012, Proc. SPIE, 8443, $84431 \mathrm{Z}$

[17] Takahashi, T., et al. 2007, PASJ, 59, 35

[18] Tanaka, Y., Inoue, H., \& Holt, S. S. 1994, PASJ, 46, L37

[19] Troja, E., Bocchino, F., \& Reale, F. 2006, ApJ, 649, 258

[20] Truemper, J. 1982, Advances in Space Research, 2, 241

[21] Yamaguchi, H., Ozawa, M., Koyama, K., et al. 2009, ApJL, 705, L6 